\title{
Solid-state batteries enter EV fray
}

\author{
By Arthur L. Robinson \\ Feature Editor Jürgen Janek
}

L ithium-ion batteries with liquid electrolytes power most of the electric vehicles $(\mathrm{EVs})$ that are widely seen as an essential step toward halting the march of global warming. But EVs currently cost significantly more while suffering from a lower driving range than gasoline- or diesel-powered vehicles. Despite significant lithium-ion battery advances in the last two decades, many in the field feel that further progress will crest in the next few years and are seeking a successor technology.

All-solid-state batteries most likely based on mobile lithium ions are an emerging option for next-generation technologies on the road to a safe, green vehicle with attractive cost and performance. What sets them apart is the use of an inorganic solid electrolyte rather than the organic liquid electrolyte embedded in a moist paste found in virtually all commercial lithium-ion batteries. One obvious virtue of a switch to the solid-state is the reduction, if not elimination, of the fire risk associated with flammable organic electrolytes when short circuits drive up the temperature.

Performance improvements, such as a higher volumetric energy density $(\mathrm{Wh} / \mathrm{L})$ to increase the driving range between charges and sufficient power density (W/L) to make energy available when needed, are just as important. Researchers also tout long cycle life and shelf life as significant improvements over today's lithium-ion batteries that degrade after a few years.

Despite these theoretical advantages, there is a long way to go before all-solid-state lithium-ion batteries begin appearing in electric vehicles. "A battery revolution is not just waiting for us around the corner," said Jürgen Janek of Justus Liebig University Giessen. "Solid-state batteries require serious efforts not only in fundamental science but also in processing technology," he said. Among the key choices to be made for any battery are the electrode and electrolyte materials. Once these are identified and workable laboratory-scale prototypes are developed, then comes the perhaps even more difficult chore of perfecting fabrication and packaging technologies that are inexpensive, rapid, and reliable on a large scale. Peter Lamp from BMW in Munich added, "It is just as important to produce a device with sufficient mechanical stability, since large volume variations in the battery materials during operation have to be taken into account. The impact of additional mechanical stress that may originate from vibrations during car operation also deserves careful evaluation."

Though not everyone agrees that the goal is realistic, auto giant Toyota is looking toward the 2020s for the introduction of vehicles powered at least, in part, by solid-state batteries. One reason is the 2012 demonstration of a prototype all-solid-state lithium-ion battery with a volumetric energy density about twice that of today's lithium-ion technology. "The first application tests with an electric kickboard, powered by the Toyota all-solidstate battery, confirm the results of our research and demonstrate the game-changing potential of this technology," said Chihiro Yada of Toyota Motor Europe, noting that candidate applications range from hybrid electric vehicles (HEVs) to plug-in hybrid electric vehicles (PHEVs) to all-electric vehicles (EVs).

Solid-state electrolytes head the list of materials options yet to be decided. Ideally, an electrolyte will have high ionic conductivity, but solid-state electrolytes historically have suffered from ionic conductivities that are orders of magnitude lower than the liquid or gel-type organic electrolytes in lithium-ion batteries. The low conductivity problem can be compounded by grain boundaries in the polycrystalline solid. Key considerations also include electrochemical stability at the operating electrode potentials (a low anode potential and higher cathode potentials are advantageous for energy density), along with ease of fabrication.

Mobile ions travel through solid electrolytes by hopping between ion lattice sites, and obtaining high conductivity at ambient temperature has been a challenge. Among the candidates are sulfide, oxide, and nitride ceramics and glasses, and even organic polymers. One breakthrough occurred in 2010 when a Japanese group led by Ryoji Kanno at the Tokyo Institute of Technology reported a lithium-germanium-phosphorous sulfide ceramic with lithium-ion conductivity at room temperature matching that of liquids, owing to channels for ion flow in the crystal structure. Balancing this success, the electrochemical stability at the interface with a lithium anode was problematic.

The Kanno group's achievement by no means rules out other materials. "Oxide-based solids with high ionic conductivity will be the leading electrolyte candidates," said Masahiro Tatsumisago of Osaka Prefecture University, a frequent collaborator with Toyota battery researchers. Jeff Sakamoto of the University of Michigan and his associates are studying one such oxide electrolyte, a lithium-lanthanum-zirconium garnet. Sakamoto explained that "Although the ionic conductivity of this compound is 
lower than that of sulfur-based electrolytes, the chemical stability against decomposition at a lithium anode is better."

With the wide range of possible electrolyte compositions, finding the optimum one is a huge challenge. In 2008, a Toyota group in Japan, for example, joined with a UK group headed by Brian Hayden at Ilika Technologies in Southampton to explore a combinatorial approach based on high-throughput vapor deposition from multiple computer-controlled sources that continuously vary the composition across a substrate. In a demonstration study of the lithium-lanthanumtitanium ternary oxide system, they were able to locate the optimum conductivity composition as well as map the distributions of phases and their composition ranges.

For a given anode material, the cathode material sets the operating voltage, and higher voltages translate to higher energy. “Toyota's prototype solid-state battery has a traditional lithium-multiple transition metaloxygen cathode that operates at $4 \mathrm{~V}$, but recent feasibility studies have used cathodes with tweaked compositions that run closer to $5 \mathrm{~V}$," said Yada. Another solid-state advantage, according to Sehee Lee of the University of Colorado at Boulder and a cofounder of the nearby battery startup Solid Power, is that "solid-state batteries offer the possibility of new chemistries that are not feasible with liquid electrolytes, such as the iron disulfide cathodes that we are working on."

Among the challenges associated with electrodes, getting lithium ions from the solid electrolyte into the cathode is often hindered by the formation of an insulating phase at the interface between the two. While placing a $\mathrm{LiNbO}_{3}$ interlayer between the electrode and electrolyte shows potential, "finding ways to mitigate the effect remains a challenge," said John Lemmon of the US Department of Energy's ARPA-E, which funds several all-solid-state battery projects with the aim of translating promising research results into technologies ready for commercialization. A similar issue arises at the anode, where lithium ions enter the electrolyte during discharge.

In conventional lithium-ion batteries, graphite has been the material of choice for the anode, though others are under development. But with "a metal anode, the lithium density at the anode would be much higher, leading to an increased energy density," said Yusheng Zhao of the University of Nevada at Las Vegas. "The problem is that with an organic liquid electrolyte, lithium dendrites would grow from the anode-electrolyte interface through the electrolyte and puncture the membrane, separating the anode and cathode compartments, leading to short-circuits and a magnified fire hazard." Solid-state lithium-ion batteries offer the option of lithium metal anodes, possibly without reintroducing a fire hazard. "A solid electrolyte might be safer," said Janek, "if it can be shown that dendrites cannot find a path along grain boundaries."

In common with lithium-ion batteries, maximizing the volume of active material and minimizing the rest is essential for making the energy density as high as possible, but it is also necessary to maximize charge transfer. A traditional linear stack comprising an anode layer (such as a lithium foil), a very thin solid-state electrolyte layer, and a cathode layer, with both electrodes in contact with a conductive material that shuttles electrons from the anode through the external circuit to the cathode particles, may not be the best structure for this. To maximize charge transfer, novel 3D battery structures, such as composite blends of electrolyte, conductive additive, and electrode material are being developed.

When it comes to fabrication, solid-state batteries require a way of thinking that departs both from today's lithium-ion batteries and from thin-film solid-state lithium-ion batteries, already an established technology for specialized military and industrial applications. Nancy Dudney of Oak Ridge National Laboratory said, "Many of these films are made using methods similar to those used to produce electronic and photovoltaic devices, but these processes are unlikely to be costeffective for the large batteries needed for EV applications."

In a presentation at the 2014 joint annual meeting of the American Ceramic Society's Glass and Optical Materials Division and the Deutsche Glastechnische Gesellschaft, Osaka's Tatsumisago and Akitoshi Hayashi concluded that realizing the ideal all-solid-state battery will require the confluence of several fabrication technologies ranging from those for film formation, particle dispersion, and mixing, to those for powders, glasses, ceramics, and polymers. High-temperature processes such as densifying powder by sintering, for example, may become important.

Should lithium all-solid-state batteries make the jump from the laboratory to the showroom floor, they are best viewed as an important step on the way to the ultimate battery. Toyota's roadmap, for example, already labels lithium-air batteries, currently behind lithium solid-state batteries on the development curve, as a possible successor during the 2030s. 